\title{
BMJ Open Acupuncture for chronic knee pain: a protocol for an updated systematic review
}

\author{
Qinhong Zhang, ${ }^{1,2}$ Jinhuan Yue, ${ }^{1}$ Zhongren Sun, ${ }^{1}$ Ying $\mathrm{Lu}^{2,3}$
}

To cite: Zhang $Q$, Yue J, Sun Z, et al. Acupuncture for chronic knee pain: a protocol for an updated systematic review. BMJ Open 2016;6: e008027. doi:10.1136/ bmjopen-2015-008027

- Prepublication history for this paper is available online. To view these files please visit the journal online (http://dx.doi.org/10.1136/ bmjopen-2015-008027).

Received 21 February 2015 Revised 9 July 2015 Accepted 16 July 2015

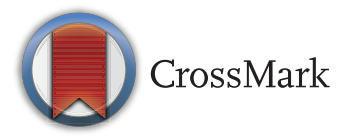

${ }^{1}$ Department of Acupuncture and Moxibustion, College of Acupuncture and Moxibustion, Heilongjiang University of Chinese Medicine, Harbin, China ${ }^{2}$ Department of Health Research and Policy, Stanford University, California, USA

${ }^{3}$ VA Palo Alto Cooperative Studies Program Coordinating Center, VA Palo Alto Health Care System, California, USA

Correspondence to Professor Zhongren Sun; sunzhongren2011@163.com and Professor Ying Lu; ylu1@stanford.edu

\section{ABSTRACT}

Introduction: The aim of this study is to evaluate the efficacy and safety of acupuncture for patients with chronic knee pain.

Methods and analysis: MEDLINE, EMBASE, CENTERAL, CINAHL and four Chinese medical databases will be searched from their inception to present. We will also manually retrieve eligible studies. Randomised controlled trials (RCTs) in which acupuncture is assessed as the sole treatment or as an adjunct treatment for chronic knee pain will be included. The primary outcome of our analysis is pain measured by the visual analogue scale (VAS), the Western Ontario and McMaster Universities Osteoarthritis Index (WOMAC) pain subscale or the 11point numeric rating scale (NRS). The secondary outcomes will include the quality of life, measured by the 36-item Short-Form Health Survey (SF-36) and adverse events. Two researchers will conduct the study selection, data extraction and quality assessment independently. Any disagreement will be resolved through discussion with a third reviewer. The Cochrane risk-of-bias criteria and the Standards for Reporting Interventions in Controlled Trials of Acupuncture (STRICTA) checklist will be used to assess the methodological quality of the trials.

Dissemination: This systematic review will assess the current evidence on acupuncture therapy for chronic knee pain. It uses aggregated published data instead of individual patient data and does not require an ethical board review and approval. The findings will be published in a peer-reviewed journal and disseminated in conference presentations. It will provide the latest analysis of the currently available evidence for acupuncture treating chronic knee pain. Trial registration number: CRD42014015514.

\section{INTRODUCTION}

Chronic knee pain affects $46.8 \%$ of older adults and is the most commonly reported cause of pain in this population, ${ }^{12}$ especially among people aged 50-69 years. ${ }^{3}{ }^{4}$ Knee pain is typically caused by knee joint osteoarthritis and frequently leads to functional limitations, which is often accompanied by psychological impairment ${ }^{5}$ and a decreased quality of life. Pharmacological therapy may offer some limited benefit for patients with chronic knee pain, but it can also cause serious adverse effects. ${ }^{6}$ Joint replacement and other surgeries are an effective way to treat patients with advanced disease. ${ }^{7}$ However, surgery may be contraindicated in patients with significant comorbidities and is quite expensive.

Acupuncture therapy involves the insertion of disposable needles into specific points on the body surface known as acupuncture points, or acupoints. ${ }^{8}$ It has a relatively high safety profile when performed by qualified acupuncturists. ${ }^{9} 10$

Acupuncture is widely used to treat a variety of conditions, such as pressure ulcers, ${ }^{11}$ neck pain, ${ }^{12}$ chronic knee pain ${ }^{13} 14$ and others. It is a popular treatment for pain associated with osteoarthritis. For example, a study reported that $61 \%$ of patients with osteoarthritis had used acupuncture in the UK. ${ }^{15}$ However, whether acupuncture actually reduces chronic knee pain remains controversial. Proponents argue that acupuncture is effective at relieving symptoms and chronic pain, while improving the quality of life. ${ }^{13} 1416$ In addition, acupuncture is associated with fewer adverse effects than conventional medical approaches for treating chronic knee pain. However, a recent study $^{17}$ in the Journal of the American Medical Association (JAMA) concluded that acupuncture negatively affected chronic knee pain, though the study had several shortcomings in its clinical design. $^{18-25}$

The efficacy of acupuncture for chronic knee pain has been evaluated in several previous systematic reviews. ${ }^{12} 1426$ All those studies failed to include all of the relevant articles published in China. ${ }^{12}{ }^{14} \quad{ }^{26}$ Furthermore, new papers have been published since those reviews. ${ }^{17}$ The most recent systematic review and meta-analysis of acupuncture for chronic knee pain found that 
acupuncture can significantly reduce pain intensity and improve functional mobility and the quality of life. ${ }^{27}$ However, this conclusion was based in part on data of pain intensity that was incorrectly pooled from four studies. In addition, the heterogeneity of the pooled data for functional mobility was excessively high. These weaknesses limit the veracity of the study conclusions. Therefore, this systematic review aims to update the previous systematic review and to further critically assess the efficacy and safety of acupuncture for chronic knee pain with the inclusion of additional studies.

\section{OBJECTIVES}

We will conduct a systematic review to assess the efficacy and safety of acupuncture for chronic knee pain. We want to establish at the study population level to determine whether real acupuncture is superior to no-acupuncture control for treating knee pain.

\section{METHODS/DESIGNS}

\section{Phase I: systematic review to identify eligible papers}

Study registration

The protocol for this systematic review is registered with PROSPERO 2014 (registration number: CRD42014015514). This protocol is conducted and reported according to the Preferred Reporting Items for Systematic Reviews and Meta-Analyses Protocols (PRISMA-P) statement guidelines, ${ }^{28}$ while our review will be operated depending on the Preferred Reporting Items for Systematic Reviews and Meta-Analyses (PRISMA) statement guidelines. ${ }^{29}$

\section{Trial eligibility criteria}

Study types

We will consider randomised controlled trials (RCTs) comparing acupuncture with non-acupuncture in patients with chronic knee pain. Non-randomised studies will be excluded.

\section{Participants}

Patients of any age, gender or ethnicity described as having chronic knee pain will be included.

\section{Sample size}

The study should have a formal sample size determination prior to the trial implementation.

\section{Interventions}

Any type of acupuncture used as the sole treatment or as a significant adjunct to other treatments for chronic knee pain will be included, such as acupuncture compared with non-acupuncture, or acupuncture combined with another intervention (non-acupuncture) compared with the other treatment alone (the same as the acupuncture group).
Outcome measures

\section{Primary outcomes}

Chronic knee pain will be assessed by the visual analogue scale (VAS) $(0-100),{ }^{30}$ the Western Ontario and McMaster Universities Osteoarthritis Index (WOMAC) score pain subscale ${ }^{3132}$ or the 11-point numeric rating scale (NRS). ${ }^{33}$

\section{Secondary outcomes}

Quality of life will be measured by the 36-item Short-Form Health Survey (SF-36). ${ }^{34}$ In addition, any adverse events will be recorded.

\section{Search methods to identify studies}

We will electronically search the following databases from their inception through present: MEDLINE, EMBASE, CENTERAL, CINAHL, the Chinese Biomedical Literature Database (CBM), the China National Knowledge Infrastructure (CNKI), VIP Information (VIP) and Wanfang Data (WAN FANG). We will also retrieve unpublished protocols and summary results through a search of the clinical trial registry at https://clinicaltrials.gov/. The provisional search strategy has been decided after discussion with all reviewers. The keywords will include 'pain', 'knee', 'knee pain', 'chronic knee pain', 'arthritis', 'osteoarthritis', 'rheumatology', 'acupuncture', 'acupuncture therapy', 'manual acupuncture', 'electroacupuncture' or 'scalp acupuncture'. The search words to be used in the Chinese databases have the same meaning as those used in the English databases. In addition, we will search the reference lists of previously published reviews related to chronic knee pain and acupuncture.

\section{Phase II: trial evaluation and data collection Study selection}

Studies will be selected independently by two reviewers (JY and QZ). Disagreements will be resolved with a third reviewer (ZS or YL) through discussion. No language restrictions will be imposed. The entire process of study selection is summarised in the PRISMA flow diagram (figure 1).

\section{Data extraction}

All included trials will be read by two reviewers ( $\mathrm{JY}$ and QZ) independently, and the data will be extracted using a predefined data extraction sheet. The extracted data will include the author, title, publication year, journal, country, participant characteristics, study size, randomisation, allocation concealment, blinding, acupuncture intervention, control intervention, main outcomes, adverse effects, follow-up, withdrawals, conflicts of interest and the Standards for Reporting Interventions in Controlled Trials of Acupuncture (STRICTA) checklist.

\section{Quality assessment}

Quality will be assessed using the Cochrane risk-of-bias tool outlined in the Cochrane Handbook for Systematic 
Figure 1 Flow diagram of the trial selection process. RCT, randomised controlled trial.
Records identified through database searching
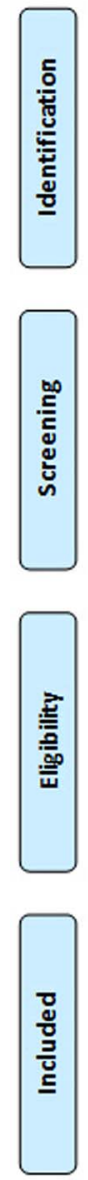

Reviews of Interventions ${ }^{35}$ and completeness of STRICTA checklist independently by two reviewers (YH and $\mathrm{ZH}$ ). All discrepancies will be resolved by discussion with the fourth author (YL).

\section{Measures of treatment effect}

For continuous outcomes such as pain scales, the mean difference (MD) with a 95\% CI will be used. Other forms of continuous data will be converted into MD values. For dichotomous data (eg, adverse events), a risk ratio (RR) with a 95\% CI will be used. Other binary data will be converted into an RR value.

\section{Unit of analysis concerns}

We will not include cluster-randomised trials and crossover studies because they lack the appropriate design for the study objectives.

\section{Missing data}

We will contact the original study authors for missing data whenever possible. Only the available data will be analysed if it is not possible to acquire the missing data. $(n=)$

Additional records identified

through other sources

Reference lists of relevant studies

or previous reviews and a Web of

Science $\operatorname{search}(n=)$

Registry at www. Clnical Trials.gov

$(n=)$

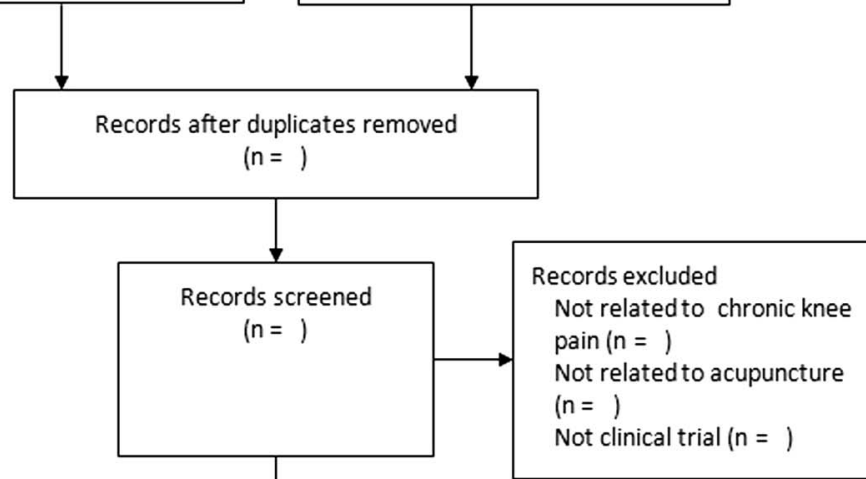

Full-text articles excluded, with reasons Not an RCT $(n=)$ RCT, but excluded because: - Wrong intervention or comparator $(\mathrm{n}=)$

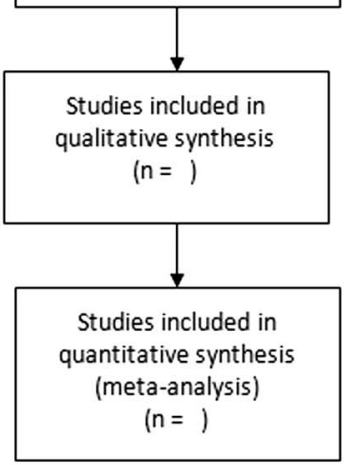

Phase III: statistical methods

Data synthesis

We will use the random-effects or fixed-effects model for the meta-analysis, if possible. Review Manager (V.5.3) software (Review Manager (RevMan) [Computer program], Version 5.3; Copenhagen: The Nordic Cochrane Centre, The Cochrane Collaboration, 2014) (The Cochrane Collaboration, Oxford, England) will be used to conduct the meta-analysis and calculate the RR with the 95\% CI for dichotomous data, and the MD with the $95 \%$ CI for continuous data. If heterogeneity is not high $\left(I^{2} \leq 50 \%\right)$, then the RR and MD will be calculated by the fixed-effects model; otherwise, the random-effects model will be used. If quantitative synthesis is not appropriate, we will report the results as the narrative description.

\section{Assessment of heterogeneity}

Heterogeneity will be investigated by the $\mathrm{I}^{2}$ and $\chi^{2}$ tests. Significant heterogeneity will be assumed if $\mathrm{I}^{2}$ is $>50 \%$, which is the cut-off point for our $\mathrm{I}^{2}$ statistical analysis. ${ }^{36}$ If significant heterogeneity is observed, then we will conduct a subgroup analysis to explore the possible causes. ${ }^{36}$ 


\section{Subgroup analysis}

Subgroup analysis will be performed to assess the heterogeneity between the studies. The analysis will include the acupuncture type (including manual acupuncture and electroacupuncture), type of control, countries and different outcomes.

\section{Sensitivity analysis}

Sensitivity analysis will be conducted by removing the impact of lower quality studies if heterogeneity remains after subgroup analysis or studies with incomplete results according to the STRICTA checklist. The meta-analysis will be repeated after lower quality studies are excluded. The results of these two meta-analyses will then be compared and discussed according to their sample size, strength of evidence and influence on the pooled effect size.

\section{Assessment of reporting biases}

If a sufficient number of included studies (at least 10 trials) are available, then the publication bias will be evaluated using funnel plots. ${ }^{37} \mathrm{We}$ will also examine impacts of possible selective reporting, reporting deviations from the original protocols, effect of protocol compliance and adherence.

\section{DISCUSSION}

Acupuncture therapy is a suggested alternative intervention to treat chronic knee pain. This systematic review will provide a detailed summary of the current evidence for the effects of acupuncture on chronic knee pain. However, this study might suffer from high heterogeneity, potential missing data and meta-biases, which may limit the quality of evidence. As our review will be based on aggregated trial data and primary focuses on the treatment effect of acupuncture, we may not have sufficient information to address efficacy at different causes of knee pain. Nonetheless, the results of this study will provide the latest analysis of the currently aggregated evidence for the efficacy of acupuncture in treating chronic knee pain, which will benefit practitioners, patients and healthcare policy-makers.

Contributors QZ, JY, ZS and YL conceived the study, designed the study protocol and drafted the manuscript. All authors contributed to the further writing of the manuscript as well as read and approved the final manuscript.

Funding The first author was supported by the Foundation of Heilongjiang University of Chinese Medicine (number 2012RCQ64) and the Foundation of Graduate Innovative Plan of Heilongjiang Province (number YJSCX2012-357HLJ).

Competing interests None declared.

Provenance and peer review Not commissioned; externally peer reviewed.

Open Access This is an Open Access article distributed in accordance with the Creative Commons Attribution Non Commercial (CC BY-NC 4.0) license, which permits others to distribute, remix, adapt, build upon this work noncommercially, and license their derivative works on different terms, provided the original work is properly cited and the use is non-commercial. See: http:// creativecommons.org/licenses/by-nc/4.0/

\section{REFERENCES}

1. Felson DT, Naimark A, Anderson J, et al. The prevalence of knee osteoarthritis in the elderly. The Framingham Osteoarthritis Study. Arthritis Rheum 1987;30:914-18.

2. Jinks $C$, Jordan $K$, Croft $P$, et al. A brief screening tool for knee pain in primary care (KNEST). 2. Results from a survey in the general population aged 50 and over. Rheumatology 2004;43:55-61.

3. Woolf AD, Pfleger B. Burden of major musculoskeletal conditions. Bull World Health Organ 2003;81:646-56.

4. Busija L, Bridgett L, Williams SRM, et al. Osteoarthritis. Best Pract Res Clin Rheumatol 2010;24:757-68.

5. Summers MN, Haley WE, Reveille JD, et al. Radiographic assessment and psychologic variables as predictors of pain and functional impairment in osteoarthritis of the knee or hip. Arthritis Rheum 1988;31:204-9.

6. Bjordal JM, Ljunggren AE, Klovning A, et al. Non-steroidal anti-inflammatory drugs, including cyclo-oxygenase-2 inhibitors, in osteoarthritis knee pain: meta-analysis of randomized placebo controlled trials. BMJ 2004;329:1317.

7. [No authors listed]. Recommendations for the medical management of osteoarthritis of the hip and knee. American College of Rheumatology Subcommittee on Osteoarthritis Guidelines. Arthritis Rheum 2000;43:1905-15.

8. VanderPloeg K, Yi X. Acupuncture in modern society. J Acup Meridian Stud 2009;2:26-33.

9. Witt CM, Pach D, Brinkhaus B, et al. Safety of acupuncture: results of a prospective observational study with 229,230 patients and introduction of a medical information and consent form. Forsch Komplementmed 2009;16:91-7.

10. Macpherson $\mathrm{H}$, Scullion $\mathrm{A}$, Thomas $\mathrm{KJ}$, et al. Patient reports of adverse events associated with acupuncture treatment: a prospective national survey. Qual Saf Health Care 2004;13:349-55.

11. Yan CL, Liu QJ, Yang $P$, et al. Clinical research on bedsores treated with fire needle therapy. Zhongguo Zhen Jiu 2010;30:819-21.

12. Fu LM, Li JT, Wu WS. Randomized controlled trials of acupuncture for neck pain: systematic review and meta-analysis. J Altern Complement Med 2009;15:133-45

13. Witt $C$, Brinkhaus $B$, Jena $S$, et al. Acupuncture in patients with osteoarthritis of the knee: a randomised trial. Lancet 2005;366:136-43.

14. Vickers AJ, Cronin AM, Maschino AC, et al. Acupuncture for chronic pain: individual patient data meta-analysis. Arch Intern Med 2012;172:1444-53.

15. Breuer $\mathrm{G}$, Orbach $\mathrm{H}$, Elkayam $\mathrm{O}$, et al. Perceived efficacy among patients of various methods of complementary alternative medicine for rheumatologic diseases. Clin Exper Rheumatol 2005;23:693-6.

16. Mavrommatis Cl, Argyra E, Vadalouka A, et al. Acupuncture as an adjunctive therapy to pharmacological treatment in patients with chronic pain due to osteoarthritis of the knee: a 3-armed, randomized, placebo-controlled trial. Pain 2012;153:1720-6.

17. Hinman RS, McCrory P, Pirotta M, et al. Acupuncture for chronic knee pain: a randomized clinical trial. JAMA 2014;312:1313-22.

18. Zhang Q, Yue J, Lu Y. Acupuncture treatment for chronic knee pain: study by Hinman et al underestimates acupuncture efficacy. Acupunct Med 2015;33:170.

19. White A, Cummings M. Acupuncture for knee osteoarthritis: study by Hinman et al represents missed opportunities. Acupunct Med 2015;33:84-6.

20. Li YM. Treating chronic knee pain with acupuncture. JAMA 2015;313:628.

21. Lao L, Yeung WF. Treating chronic knee pain with acupuncture. JAMA 2015;313:627-8.

22. Fleckenstein J, Banzer W. Treating chronic knee pain with acupuncture. JAMA 2015;313:627.

23. $\mathrm{He} \mathrm{H}$. Treating chronic knee pain with acupuncture. JAMA 2015;313:626.

24. Baxter GD, Tumilty S. Treating chronic knee pain with acupuncture. JAMA 2015;313:626-7.

25. Fan AY. The methodology flaws in Hinman's acupuncture clinical trial part I: design and results interpretation. J Integr Med 2015;13:65-8.

26. White A, Foster NE, Cummings $M$, et al. Acupuncture treatment for chronic knee pain: a systematic review. Rheumatology (Oxford) 2007;46:384-90.

27. Manyanga T, Froese M, Zarychanski R, et al. Pain management with acupuncture in osteoarthritis: a systematic review and meta-analysis. BMC Complement Altern Med 2014;14:312.

28. Shamseer L, Moher D, Clarke M, et al. Preferred reporting items for systematic review and meta-analysis protocols (PRISMA-P) 2015: elaboration and explanation. BMJ 2015;349:g7647.

29. Liberati A, Altman DG, Tetzlaff J, et al. The PRISMA statement for reporting systematic reviews and meta-analyses of studies that 
evaluate health care interventions: explanation and elaboration. Ann Intern Med 2009;151:W65-94.

30. Crichton N. Information point: visual analogue scale (VAS). J Clin Nurs 2001;10:697-706.

31. Theiler R, Spielberger J, Bischoff HA, et al. Clinical evaluation of the WOMAC 3.0 OA Index in numeric rating scale format using a computerized touch screen version. Osteoarthritis Cartilage 2002;10:479-81.

32. Bellamy N, Campbell J, Stevens J, et al. Validation study of a computerized version of the Western Ontario and McMaster Universities VA3.0 Osteoarthritis Index. J Rheumatol 1997;24:2413-5.

33. Farrar JT, Young JP Jr, LaMoreaux L, et al. Clinical importance of changes in chronic pain intensity measured on an 11-point numerical pain rating scale. Pain 2001;94:149-58.

34. Brazier JE, Harper R, Jones NM, et al. Validating the SF-36 health survey questionnaire: new outcome measure for primary care. BMJ 1992;305:160-4.
35. Higgins JPT, Altman DG, Sterne JAC on behalf of the Cochrane Statistical Methods Group and the Cochrane Bias Methods Group. Chapter 8: assessing risk of bias in included studies. In: Higgins JPT, Green S, eds. Cochrane handbook for systematic reviews of interventions version 5.1.0 (updated March 2011). The Cochrane Collaboration, 2011. http://www.cochrane-handbook.org (accessed 4 Feb 2015).

36. Deeks JJ, Higgins JPT, Altman DG. Chapter 9: analysing data and undertaking meta-analyses. In: Higgins JPT, Green S, eds. Cochrane handbook for systematic reviews of interventions version 5.1.0 (updated March 2011). The Cochrane Collaboration, 2011. http://www.cochrane-handbook.org

37. Sterne JAC, Egger M, Moher D. Chapter 10: addressing reporting biases. In: Higgins JPT, Green S, eds. Cochrane handbook for systematic reviews of interventions version 510 (updated March 2011). The Cochrane Collaboration, 2011. http://www. cochrane-handbook.org 